\title{
Paul Klimpel
}

\section{Neue Chancen für das kulturelle Erbe A fresh chance for cultural heritage}

\author{
https://doi.org/10.1515/bd-2020-0070
}

\begin{abstract}
Kulturinstitutionen müssen zahlreiche rechtliche Hindernisse überwinden, um kulturelles Erbe Online zu präsentieren. Das Urheberrecht erweist sich häufig als ein kaum lösbares Problem, insbesondere bei Zeugnissen sozialer Bewegungen wie der Frauenbewegung oder der Friedensbewegung, die niemals kommerziell vermarktet wurden. Die Klärung des urheberrechtlichen Stauts jedes einzelnen Objekts ist bei Massendigitalisierung kaum möglich. Die DSM-Richtline der EU wird die Situation verbessern, wobei einiges von den Details der Umsetzung abhängt. Dies gilt insbesondere für die Rolle der Verwertungsgesellschaften.
\end{abstract}

Schlüsselwörter: Kulturelles Erbe, Rechteklärung, vergriffene Werke

\begin{abstract}
Cultural Institutions still encounter many legal obstacles if they want to present cultural heritage works online. Copyright laws are often an unsolvable problem, especially with testimonies of social movements such as women's liberation and the peace movement, which have never been commercialised. Rights clearance of the copyright status of individual objects is nearly impossible in mass digitisation contexts. The EU directive on Copyright in the Digital Single Market will help to improve the situation, although proper implementation will depend on important particulars such as the role of collecting societies and royalties organisations.
\end{abstract}

Keywords: Cultural heritage, rights clearance, out-of-print works

Das Bild von der Vergangenheit wird heute durch das geprägt, was von ihr online zugänglich ist. Der erste Zugang zu unserer Geschichte ist heute das, was mit ein paar Mausklicks erreichbar ist. Erst dann folgt für die große Mehrheit die Vertiefung an den Orten, die extra dafür unterhalten werden. Kaum jemand besucht noch ein Archiv, ein Museum oder eine Bibliothek, ohne sich zuvor online zu informieren.

Dr.Paul Klimpel post@paulklimpel.de 
Doch immer wieder mussten Fachleute aus diesen Einrichtungen in den letzten Jahren warnend die Befürchtung äußern, das Zwanzigste Jahrhundert könnte im Internet ein schwarzes Loch bleiben. Denn wichtige Zeugnisse dieser Zeit können von ihnen aufgrund der komplizierten Rechtslage dort eben nicht zugänglich gemacht werden. Bevor Kulturinstitutionen ihre Bestände online präsentieren können, müssen sie klären, ob diese Bestände noch urheberrechtlich geschützt sind, wer die Rechteinhaber sind und ob diese dann zustimmen, die Digitalisate online zugänglich zu machen. Das war und ist ein sehr kompliziertes Unterfangen, gerade bei älteren Werken. Da der Schutz, den das Urheberrecht gewährte, mit 70 Jahren nach Tod des Urhebers sehr viel länger dauert als die gewöhnlichen kommerziellen Verwertungen, ist häufig nicht mehr feststellbar, wer welche Rechte an solchen Zeugnissen hat.

Besonders schwierig ist die Lage bei Objekten, die niemals Teil der kulturellen Verwertung waren, sondern den Alltag oder auch soziale Bewegungen dokumentieren, wie etwa ein Flugblatt aus der Zeit der Studentenproteste, eine Broschüre der Schwulenbewegung, ein Aufruf der Friedensbewegung, Texte der Umweltbewegung oder eine Deklaration der Frauenbewegung. Diese zeitgeschichtlichen Zeugnisse sind oft am Rande dessen, was rechtlich gesehen als „Werk“ urheberrechtlich geschützt ist. Denn rechtlich ist das Verständnis dessen, was als Werk gilt und damit Urheberrechtsschutz genießt, sehr weitgehend. Während im allgemeinen Sprachgebrauch mit dem Begriff „Werk“ Romane, Gemälde oder Symphonien assoziiert werden, können rechtlich (nach dem Konzept der „kleinen Münze“) auch kurze Textpassagen als Werke gelten. Insgesamt ist die Schwelle der sogenannten „Schöpfungshöhe“, also der Schwelle, ab wann ein Werk urheberrechtlichen Schutz genießt, nach heutigem Verständnis sehr niedrig. Unter Juristen ist vieles umstritten, auch die Rechtsprechung ist nicht immer einheitlich. So erkannten manche Gerichte bereits Datenschutzerklärungen oder Gebrauchsanweisungen urheberrechtlichen Schutz zu, während andere die Schutzfähigkeit von Formularverträgen verneinten.

Besonders gravierend ist, dass grundsätzlich jedes Foto urheberrechtlich geschützt ist, wobei das Gesetz hier Unterschiede macht, die allerdings zu Abgrenzungsschwierigkeiten führen. Gemeint ist die Unterscheidung zwischen einem fotografischen „Werk“ - dann gilt der Schutz, wie bei allen Werken, bis 70 Jahre nach Tod des Urhebers, - und einem simplen „Knipsbild“, das lediglich 50 Jahre lang geschützt wird.

Wer einmal einen Blick in die Depots von Archiven oder Museen geworfen hat und die Kartons mit Fotos, Akten, Broschüren, Erklärungen und Flugblättern gesehen hat, dem wird schnell klar, dass die Klärung des urheberrechtlichen Status jedes einzelnen dieser Objekte ein nahezu unmögliches Unterfangen ist. 
Bisher waren die gesetzgeberischen Bemühungen, diese Situation zu verbessern, sehr zaghaft. 2013 trat eine Richtlinie in Kraft, die die Nutzung ,verwaister“ Werke ermöglichen sollte. Als „verwaist“ gelten solche Werke, deren Rechteinhaber trotz sorgfältiger Suche nicht identifizierbar oder lokalisierbar sind. Damit wollte die EU dem Bestreben, europäische Kultur online verfügbar zu machen - dem unter anderem auch das gemeinsame Portal „Europeana“ dient eine bessere Grundlage geben. Allerdings hat diese Richtlinie den Praxistest nicht bestanden und gilt gemeinhin als gescheitert.

Warum? Kaum ein Museum ließ sich auf den aufwändigen, personalintensiven und kostspieligen Prozess einer „sorgfältigen Suche“ ein, die die Richtlinie einfordert - zumal diese Suche dann auch noch dokumentiert werden muss. Hinzu kommt, dass sich diese Investitionen in die Rechteklärung, wie auch die in die Digitalisierung und Online-Stellung selbst, als vergeblich herausstellen können - immer dann, wenn sich Rechteinhaber dann doch noch melden und verlangen, die Online-Präsentation ihrer Werke zu beenden. Und nicht nur das, es besteht sogar das Risiko, dass die zumeist finanziell klammen Kulturinstitutionen für vergangene Nutzungen eine Entschädigung zahlen müssen.

Unter nahezu allen Fachleuten herrscht Einigkeit, dass die eben beschriebene, derzeit geltende Rechtslage unbefriedigend ist. In ihrer „Hamburger Note“ haben 2015 die führenden Rechtswissenschaftlerinnen und Rechtswissenschaftler sowie die Leiterinnen und Leiter der wichtigsten Einrichtungen des kulturellen Erbes festgehalten, dass „die Chancen der Digitalisierung nur genutzt werden können bei gesetzlichen Rahmenbedingungen, die für alle öffentlichen Gedächtnisinstitutionen eine rechtliche Einzelfallprüfung entbehrlich machen“ und eine Sichtbarmachung von Beständen im Internet grundsätzlich ermöglichen. In ihrer Erklärung warnen sie: „Gelingt es nicht, die rechtlichen Rahmenbedingungen entsprechend weiter zu entwickeln, können wichtige Zeugnisse des kulturellen Erbes aus Gründen der rechtlichen Unsicherheit nicht digital genutzt werden. Dadurch droht eine bedenkliche Verzerrung unseres Geschichtsbildes. Auch werden weiter erhebliche öffentliche Ressourcen für die Klärung von Rechtsfragen aufgewendet, die den Gedächtnisinstitutionen fehlen und die auch nicht den Urhebern zugutekommen.“

Parallel zum Inkrafttreten der untauglichen Bestimmungen $\mathrm{zu}$ den verwaisten Werken ist der deutsche Gesetzgeber einen Weg gegangen, der sich als zukunftsweisender erwiesen hat. Er hat es ermöglicht, dass Verwertungsgesellschaften vergriffene Werke lizenzieren können, damit diese dann von Kulturinstitutionen digitalisiert und online gestellt werden können. Als „vergriffen“ gelten Werke, die nicht mehr lieferbar sind, etwa weil die Rechteinhaber ihre Produktion einstellten. Die Deutsche Nationalbibliothek (DNB) und die Verwertungsgesellschaft (VG) Wort nutzten diese Möglichkeit und schufen einen von nahezu allen 
Beteiligten einhellig gerühmten Lizenzierungsservice für vergriffene Bücher, die vor 1966 erschienen sind. Eine Bibliothek beziehungsweise eine andere Kultureinrichtung, die ein solches Buch digitalisieren will, meldet es bei dem von der DNB betriebenen Lizenzierungsservice an. Dieser gleicht es automatisch mit den Verzeichnissen des Buchhandels ab. Sofern das Buch tatsächlich nicht mehr lieferbar ist, übermittelt die DNB einen Lizenzantrag an die VG Wort. Sobald die VG Wort eine solche Lizenz vergibt, wird das Werk auch in das Verzeichnis vergriffener Werke beim Patent- und Markenamt aufgenommen. Die Lizenzgebühren für dieses Prozedere sind überschaubar: bei Büchern vor 1920 liegen sie bei 5 Euro, für solche, die zwischen 1921 und 1945 erschienen sind, bei 10 Euro und für zwischen 1946 bis 1965 erschienene Bücher sind 15 Euro zu zahlen. Im Gegenzug fällt der gesamte Aufwand weg, der sonst für die Recherchen nach den Rechteinhabern notwendig wäre, um die Regelungen für verwaiste Werke zu befolgen. Kein Wunder also, dass auch verwaiste Werke, für die nach der dafür geschaffenen Richtlinie gar keine Lizenzgebühr bezahlt werden muss, in der Praxis immer öfter über die Vorschriften für vergriffene Werke lizenziert werden. Denn ein verwaistes Werk ist immer auch ein vergriffenes Werk.

Bibliotheken, Archiven und Museen drängen schon lange darauf, diese erfolgreiche Regelung weiter auszuweiten, zum Beispiel auf Zeitschriften und Zeitungen, auf Sammelbände und auf jüngere Publikationen. Das bisherige Zögern der VG Wort, sich darauf einzulassen, hatte auch mit einer gewissen rechtlichen Unsicherheit zu tun. Sie war sich nicht sicher, ob dieser „deutsche Weg“ bei den vergriffenen Werken europarechtlich zulässig sei. Doch diese Bedenken sind nunmehr ausgeräumt, hat doch der europäische Gesetzgeber in der Urheberrechtsrichtlinie, die er im Frühjahr 2019 beschlossen hat, eine entsprechende Vorgabe für vergriffene Werke formuliert. Deutschland muss diese Richtlinie nun bis Frühjahr 2021 umsetzen. Dabei unterscheidet sich nicht nur das Prozedere, sondern die europäischen Regeln gehen über das hinaus, was bisher in Deutschland möglich ist.

So sollen als „vergriffene Werke“ auch solche gelten, die „ursprünglich nicht für gewerbliche Zwecke gedacht waren oder niemals gewerblich genutzt wurden“ (siehe Erwägungsgrund 30 der EU-Richtlinie). Damit könnten die Gedächtnisinstitutionen endlich auch die oben beschriebenen Zeugnisse der sozialen Bewegungen online zugänglich machen, bei denen dies bisher nicht möglich war. Doch wie so häufig, steckt der Teufel auch hier im Detail.

Die Richtlinie sieht vor, dass bei vergriffenen Werken eine Lizenzierung durch Verwertungsgesellschaften erfolgen soll, sofern ,die Verwertungsgesellschaft aufgrund ihrer Mandate ausreichend repräsentativ für die Rechteinhaber der einschlägigen Art von Werken oder sonstigen Schutzgegenständen ...“ ist. Ist dies nicht der Fall, sollen vergriffene Werke gleichwohl online gestellt werden dürfen - 
dann aber nicht aufgrund einer Lizenz, sondern aufgrund einer vergütungsfreien gesetzlichen Erlaubnis.

Nun hat Deutschland ein recht ausdifferenziertes System von 13 Verwertungsgesellschaften, die beispielsweise für Werke der Musik, der bildenden Künste, Text- und Sprachwerke und Weiteres zuständig sind. Trotzdem erscheint fraglich, ob von diesen 13 tatsächlich alles abgedeckt ist, was an urheberrechtlich geschützten Werken entsteht. Ohne Frage wird die große Mehrzahl der Autoren, die Bücher veröffentlichen, der VG Wort beitreten. Aber wird dies auch ein schwuler Aktivist tun, der 1981 einen Text gegen den damaligen Paragrafen 175 Strafgesetzbuch verfasst hat? Oder die Feministin, die ein Flugblatt gegen den Paragrafen 218 Strafgesetzbuch verfasst hat? Oder die Umweltschützerin, die einen aufrüttelnden Appell an die Politik wegen des Waldsterbens verfasst hat? Oder der APO Aktivist, der eine Broschüre über den urbanen Klassenkampf geschrieben hat? Es erscheint abwegig, die VG Wort auch für solche Texte für repräsentativ $\mathrm{zu}$ halten. Gleiches gilt für die Millionen privaten Fotos, die möglicherweise kulturgeschichtlich interessant sind, deren Erzeuger deshalb aber sicher nicht in die VG Bild-Kunst eingetreten sind.

Sieht man einmal von der Frage ab, ob die Verwertungsgesellschaften, die im Wesentlichen jene vertreten, die sich professionell an dem kulturwirtschaftlichen Prozess beteiligen, überhaupt repräsentativ für solche sind, die sich in gesellschaftspolitischen Bewegungen engagierten, so spricht auch ein anderer Grund dafür, hier eine gesetzliche Erlaubnis greifen zu lassen. Denn der Aufbau einer Infrastruktur zur Lizenzierung von solchen Werken aus dem politischen Aktivismus oder dem Privatbereich liegt nicht im Interesse der Verwertungsgesellschaften. Eine solche Infrastruktur ist teuer. Selbst wenn bei einer Lizenzierung nur der Verwaltungsaufwand bezahlt werden müsste, wäre die Lizenzgebühr für ein Flugblatt unangemessen teuer - verglichen damit, was für die Lizenzierung von Büchern bezahlt wird, die ja immerhin einmal wirtschaftlich verwertet wurden. Das heißt, die Verwertungsgesellschaften würden hier draufzahlen - und das wäre gewiss nicht im Interesse ihrer Mitglieder sowie der ihnen angeschlossenen Wahrnehmungsberechtigten.

Es muss also darum gehen, bei der Umsetzung der EU-Richtlinie in deutsches Urheberrecht eine Abgrenzung zu finden und ins Gesetz zu schreiben. Das Gesetz muss unterscheiden zwischen vergriffenen Werken, die durch Verwertungsgesellschaften lizenziert werden - also jenen, die kommerziell verwertet wurden und für die die Verwertungsgesellschaften ausreichend repräsentativ sind - und solchen Werken, die sich auf der Grundlage einer gesetzlichen Erlaubnis vergütungsfrei nutzen lassen. Diese Grenze zu ziehen, ist keine ganz leichte Aufgabe. Erschwerend kommt hinzu, dass durch die vergangenen zehn Jahre des Urheberrechtsdiskurses verhärtete Fronten entstanden und den Verwertungsgesellschaf- 
ten vergütungsfreie Erlaubnisse - wie sie die Richtlinie vorsieht - suspekt sind. Doch hier gilt, dass alle Beteiligten pragmatisch und konstruktiv gemeinsam nach einer Abgrenzung suchen - auch um die Verwertungsgesellschaften davon zu entlasten, zukünftig zu einer Lizenzierungsagentur für jedes urheberrechtlich geschützte private oder politische Zeugnis zu werden. Verwertungsgesellschaften werden für die Vertretung der Interessen der professionell am Kulturbetrieb Beteiligten benötigt und sollten ihre Energien nicht für Materialien vergeuden, die in einem ganz anderen Zusammenhang entstanden sind.

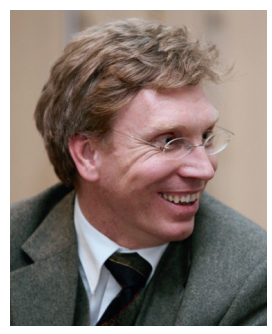

\section{Dr. Paul Klimpel}

Dieffenbachstr. 19

10967 Berlin

Deutschland

E-Mail: post@paulklimpel.de 